\title{
$\therefore$ XTENDEDS \\ MAGNETICALLY ACTUATED PROPELLANT ORIENTATION, CONTROLLING FLUIDS IN A LOW-GRAVITY ENVIRONMENT
}

\author{
By James J. Martin and James B. Holt \\ Space Transportation Directorate \\ Marshall Space Flight Center, MSFC Alabama
}

\section{Background}

Cryogenic fluid management (CFM) is a technology area common to virtually every space transportation propulsion concept envisioned. Storage, supply, transfer and handling of sub-critical cryogenic fluids are basic capabilities that have long been needed by multiple programs and the need is expected to continue in the future. The use of magnetic fields provides another method, which could replace or augment current/traditional approaches, potentially simplifying vehicle operational constraints.

The magnetically actuated propellant orientation (MAPO) program effort focused on the use of magnetic fields to control fluid motion as it relates to positioning (i.e. orientation and acquisition) of a paramagnetic substance such as $\mathrm{LO}_{2}$. Current CFM stateof-the-art systems used to control and acquire propellant in low gravity environments rely on liquid surface tension devices which employ vanes, fine screen mesh channels and baskets. These devices trap and direct propellant to areas where it's needed and have been used routinely with storable (non-cryogenic) propellants. However, almost no data exists regarding their operation in cryogenics and the use of such devices confronts designers with a multitude of significant technology issues. Typical problems include a sensitivity to screen dry out (due to thermal loads and pressurant gas) and momentary adverse accelerations (generated from either internal or external sources). Any of these problems can potentially cause the acquisition systems to ingest or develop vapor and fail.

The use of lightweight high field strength magnets may offer a valuable means of augmenting traditional systems potentially mitigating or at least easing operational requirements. Two potential uses of magnetic fields include: 1) strategically positioning magnets to keep vent ports clear of liquid (enabling low $G$ vented fill operations), and 2) placing magnets in the center or around the walls of the tank to create an insulating vapor pocket (between the liquid and the tank wall) which could effectively lower heat transfer to the liquid (enabling increased storage time). 


\title{
Objective
}

The focus of this test program was to produce experimental data regarding the interaction between a magnetic field and ferromagnetic fluid, and use the data to verify a modified computational fluid dynamics (CFD) code called CFX-4. The verified CFD model which could then be used to predict motion for other fluids, magnetic field distributions, hardware geometries, and imposed accelerations.

\begin{abstract}
Approach
Experimental hardware was fabricated and tested with fluids including a nonmagnetic water baseline and several magnetic solutions of varying strength. With the intent of making this test program simple from the standpoint of safety and handling, a commercially available water based ferromagnetic material was selected as the magnetic test fluid. Experimental data was collected in the form of video tape and accelerometer readings aboard the NASA Reduced-Gravity Workshop, a KC-135A aircraft.

\section{Test Hardware Description}

The MAPO test hardware, illustrated in figure 1 was designed, fabricated and assembled at the Marshall Space Flight Center (MSFC). This hardware package consisted of an aluminum tubular frame providing structural support for components and handrails on all sides to ease handling. The hardware had an overall envelope of 52-inch length $x 23$ inch width $\times 16$-inch height and a flight weight of approximately 150 pounds. The size and weight were kept at a minimum in an effort to maximize the amount of free float time. The package also contained its own power and lighting sources so that no connections to the aircraft were required. The components contained within the frame structure consisted of the test tank, a 6000 gauss rare earth magnet, video camera, a 3-axis accelerometer package and a laptop computer. For alt testing the tank was filled to approximately $50 \%$.
\end{abstract}

Figure 1. MAPO Test Package

\section{Test Fluid Description}

A commercial company, Ferrofluidics Corporation, manufactured the water-based ferromagnetic fluid used in these tests. This fluid selection enabled simplification of the overall program safety requirements. Safety requirements would have been significant had a kerosene-based simulant or actual oxygen been used. The ferromagnetic material was a colloidal dispersion of very fine iron oxide particles within a water base. The main 
drawback was its very dark nature and a tendency to coat surfaces rendering them opaque, an undesirable effect for systems requiring visual observation. The ferromagnetic solution, designated EMG-805, was diluted with distilled water for testing at ratios of 10:1, 30:1 and 133:1 by volume.

\section{Flight Test Environment}

All testing was performed aboard the NASA KC-135 Reduced Gravity Workshop operated by JSC out of Ellington Field in Houston Texas. The test hardware was loaded onto the aircraft and secured with several cargo straps and used approximately $1 / 3$ of the aircraft open. The large float zone provided the space necessary for maximizing the low gravity float time (before the test package drifted into the aircraft walls. Also, since there is a tendency for free floating articles to migrate rearward during a free float, the rear position assisted in keeping the test package away from other experiments. The typical duration of the low gravity float interval (or parabola) flown by the aircraft was approximately 25 seconds. However, the usable portion of the interval was typically much shorter with time consumed by the test package transition and release process. The MAPO hardware was tested in three different orientations used to provide an initial fluid position prior to the low gravity test interval.

\section{Numerical Modeling}

The use of a magnetic field provides an additional body force, Pondermotive, which can be written as

$$
\vec{F}_{M}=\mu_{0}(\vec{M} \cdot \nabla) \vec{H}
$$

where

$\mu_{0}$ - permeability of free space

$\vec{M}$ - magnetization

$\vec{H}$ - magnetic intensity

For paramagnetism and ferromagnetism the magnetization vector $\vec{M}$ is parallel to the external field that causes it. Furthermore, the magnetization $\vec{M}$ is proportional to the magnetic intensity $\vec{H}$ for paramagnetic and diamagnetic fluids. Assuming the fluid is electrically nonconducting, the displacement current is negligible and the fluid is linear in the magnetic sense, Equation (1) can be written as

$$
\vec{F}_{M}=\mu_{0} \chi \quad H^{\nabla} \mathrm{H}
$$

where

$\chi$ - magnetic susceptibility 
$\mathrm{H}$ - magnitude of the magnetic intensity

With the above assumptions the relation for the magnetic field strength and the magnetic field intensity can be written as

$$
\mathrm{B}=\mu_{0}(1+\chi) \mathrm{H}
$$

The measured magnetic parameters for the MAPO experiment included the magnetic field strength B, magnetic field direction and the ferromagnetic solution magnetic susceptibility $\chi$. These values were used in Equation (2) to compute the magnetic force on the fluid throughout the volume of the MAPO test tank.

The Computational Fluid Dynamics (CFD) code selected for this project is a commercially available product called CFX -4 distributed by AEA Technology. The current MAPO model utilized the following CFX-4 options: surface tension model, incompressible, two phase (liquid/gas), laminar, isothermal, transient, buoyant flow. The grid structure for the MAPO test tank analyses, consisted of five blocks with a total of 40,700 volumes. The grid is primarily uniform with some clustering near the walls of the tank.

\section{Test Results}

A total of 111 low gravity float tests were performed during the three test days aboard the KC-135. After reviewing the video and accelerometer data nine tests were selected as candidates to pursue further with computational modeling.

The video and accelerometer data recorded during flight testing was transferred to the computer for processing so that it could be compared to the predictions from the CFD model. A baseline water test was performed showing the motion of a non-magnetic fluid (water) in the presence of a magnetic field. This test is illustrated by the image sequence depicted in figures 2. As expected, both tests show no tendency for the water to be attracted toward the magnet, however, they provided an excellent demonstration of the CFX-4 code's ability to model the basic motion of the liquid and its vapor interface. 

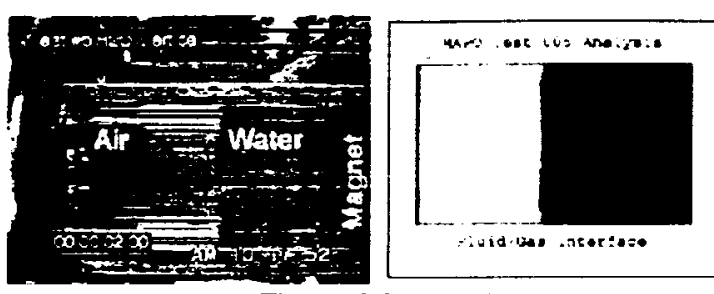

Time $=2.0$ seconds
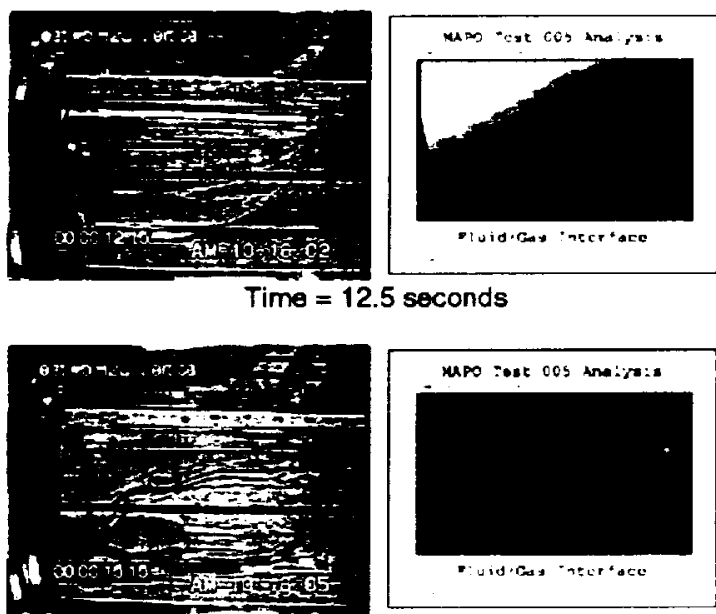

Time $=15.5$ seconds
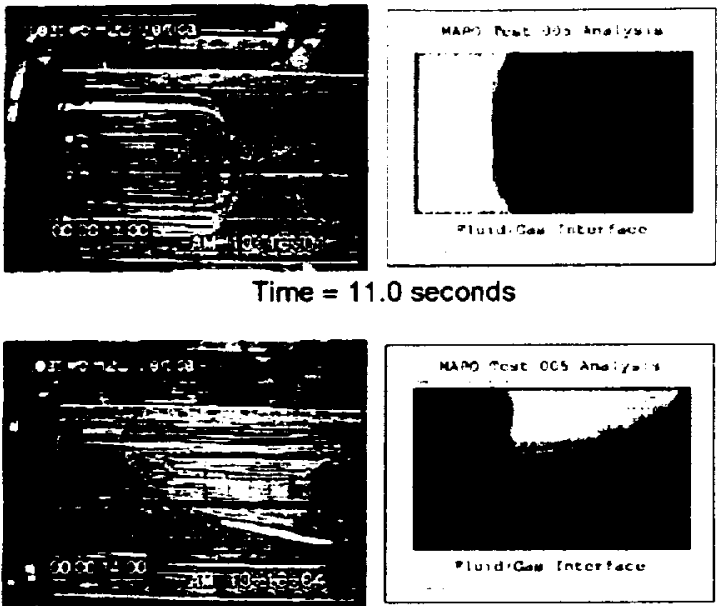

$\operatorname{Time}=14.0$ seconds

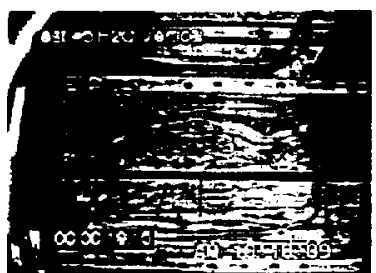

$\operatorname{Time}=19.5$ seconds

Figure 2 Baseline Water Test Image Frames

The test sequence shown in figure 3, employed a ferromagnetic solution. The mixture ratio selected was 30 parts water to 1 part ferromagnetic solution (30:1). As expected, the fluid moved toward the bottom of the tank (magnet side) when the low gravity environment was introduced. The release and transition into the low gravity environment generated fluid slosh, which moved slowly back and forth between the tank ends coating almost the complete tank surface. However, a small region of the tank air space was left uncoated and visible, indicating that the fluid has moved toward the magnet end of the test tank. At 23.5 seconds the test package was intercepted and the test concluded.

The CFD model shows excellent correspondence with the visible test data, predicting bulk fluid motion toward the magnet end of the tank where it settles. The predicted fluid surface shape tracks the test data very well to the 13-second image frame where the fluid coating effect begins to obscure the view. The image sequence illustrated by the model coated surface shows excellent correspondence with the experimental data, capturing an evolution of the uncoated visible area in the tank air space almost identical to the pattern recorded in the test data. 

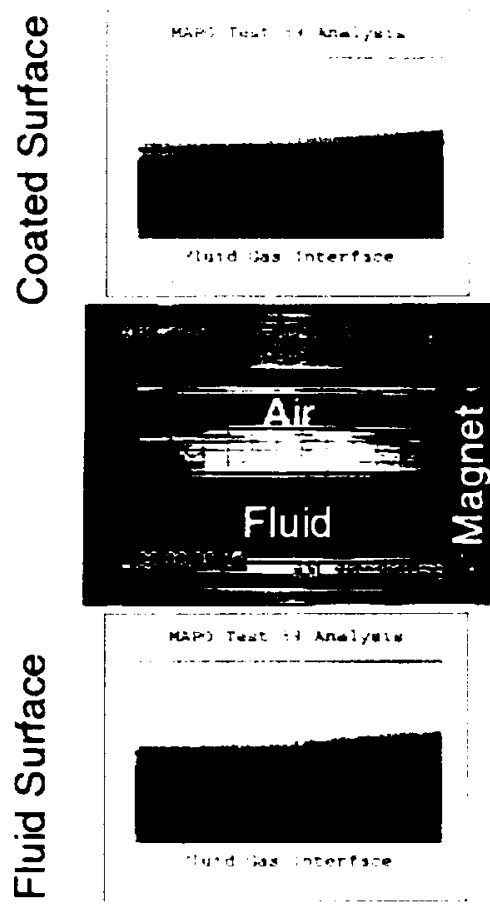

Time $=0.5$ seconds

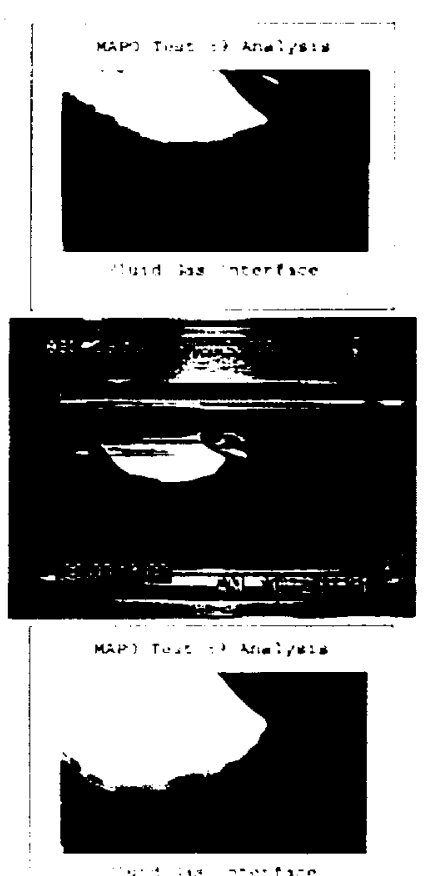

Time $=13.0$ seconds

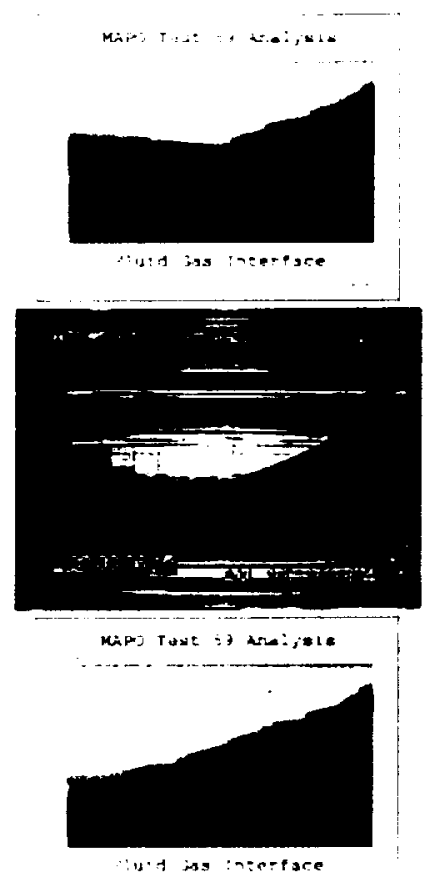

Time $=6.5$ seconds

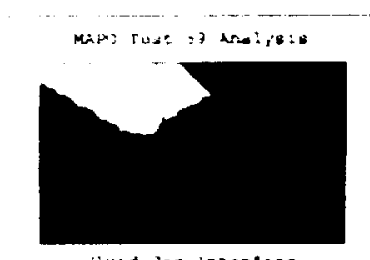

711:d bas ancepace
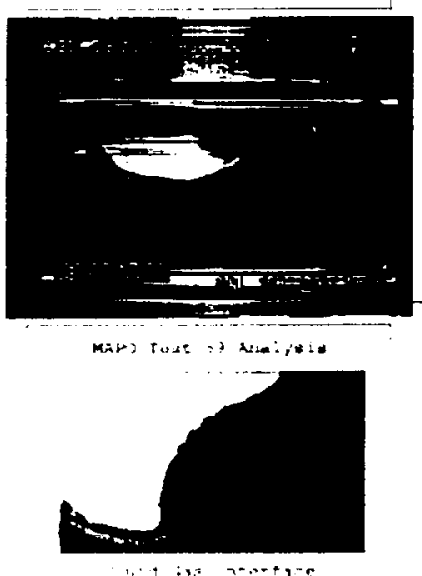

Time $=17.0$ seconds

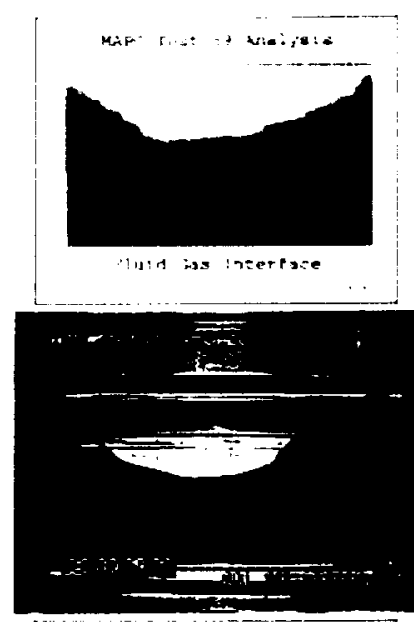

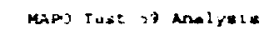

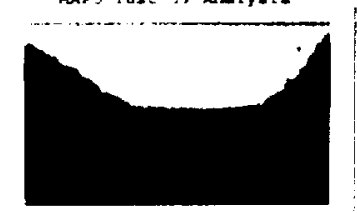

$\because$ atd bis incerface

Time $=9.0$ seconds
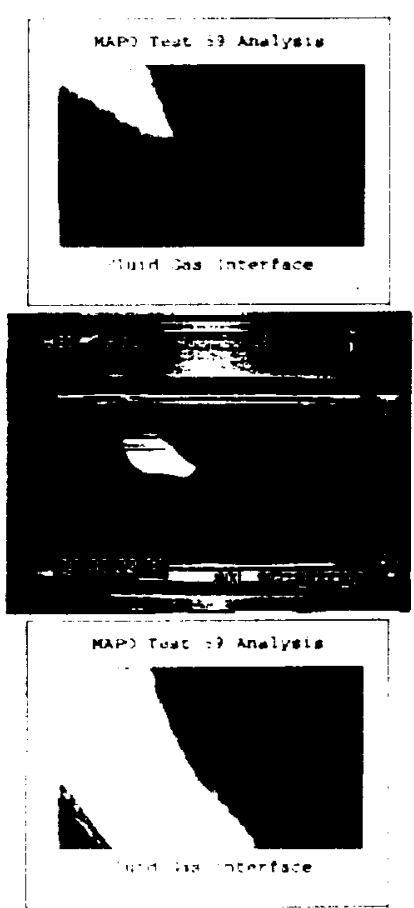

Time $=22.0$ seconds

Figure 3 Mixture 30:1 Test Frames 


\section{Discussion}

The results presented in the previous section showed that the CFD model was very successful in tracking the fluid motion across a range of test conditions. These initial tests have built confidence in the ability of the CFD model and its potential for use in modeling other fluids such as liquid oxygen. However, it was evident that the use of ferromagnetic solutions limited the quantity and quality of the data recorded because the solution would coat the tank walls prohibiting continuous and precise viewing of the fluid motion. Also, the test environment aboard the $\mathrm{KCl} 35$ was highly variable, full of unpredictable movements and vibrations resulting in significant agitation during the package release phase creating significant fluid slosh. Often the agitation was sufficient to coat the tank and effectively terminate the test before the low gravity float even began.

A simulation was performed using the test tank geometry and the properties of LO2. This simulation is illustrated in figure 4 and predicts the movement generated by several different magnetic field strengths. 

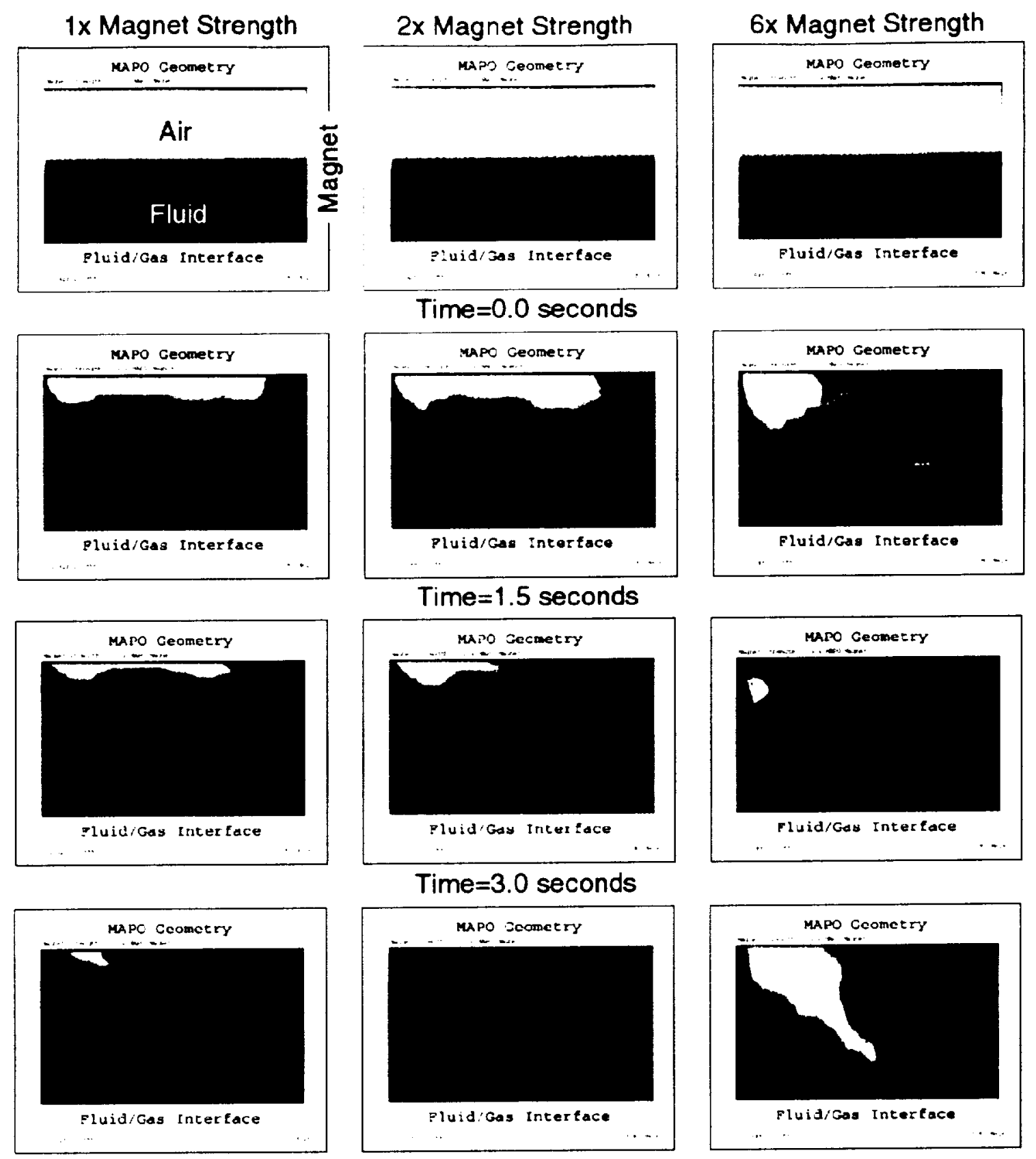

\section{Time $=3.0$ seconds}

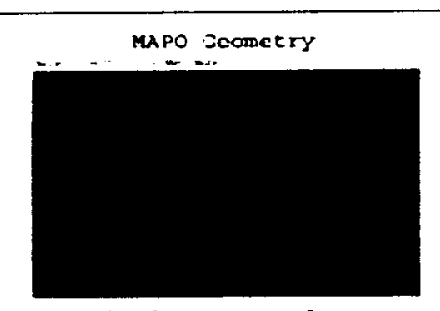

Pluidioas Intertace

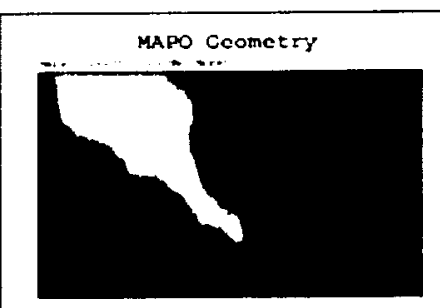

Fluid/Gas Interface

Time $=6.0$ seconds

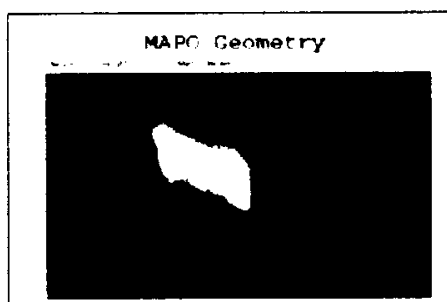

Pluidlas interface

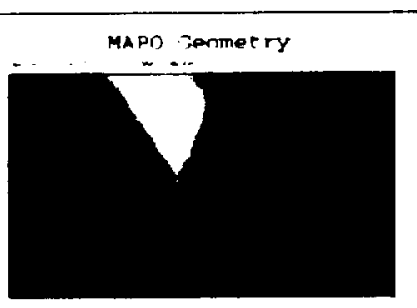

Fluid: Sas Intertace

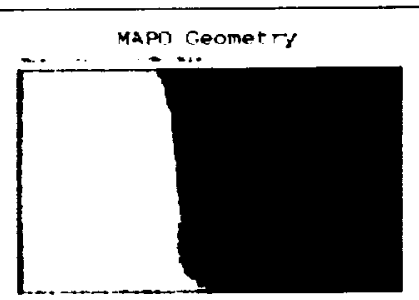

Fluid/as Interface

Time $=14.0$ seconds

Figure $4 \mathrm{LO}_{2}$ Motion Simulation with 1x, 2x and 6x Baseline Magnetic Field 
It is obvious that the $6 \mathrm{x}$ magnetic field rapidly settles the fluid against the bottom of the tank. The $2 \mathrm{x}$ field case also settles a majority of the $\mathrm{LO}_{2}$ during the simulation. The results for the $1 \mathrm{x}$ field simulation are similar to those of the $2 \mathrm{x}$ case however additional time would be required to ascertain how much more if any fluid would transfer to the bottom of the tank.

\section{Conclusions}

The use of water based ferromagnetic material and the KC135 provided a good method for quickly obtaining low cost and non-hazardous experimental data regarding the effect of magnetic fields on fluids. However, due to the dark opaque nature of the ferromagnetic mixture, fluid motion was many times obscured after the test tank walls became coated. Hence, considerable care was required in the handling and release techniques used to free float the test package. The combination of difficult fluid visualization and unpredictable aircraft motion required that a large number of tests be performed, from which only nine were acceptable for further investigation. This program should be viewed as a first step, and follow-on activities in paramagnetic fluid behavior should employ $\mathrm{LO}_{2}$ eliminating uncertainties associated with fluid visibility.

The CFD model (CFX-4 by AEA Technologies), modified to include magnetic body forces, performed very well overall in simulating the nine experimental tests. The model required no special case by case "tweaking" and was given input, which included: fluid properties, magnetic field distribution and test package acceleration profile. With the initial success of the CFD model, a simulation with $\mathrm{LO}_{2}$ was performed for various magnetic field strengths. These results showed that although the $\mathrm{lx}$ baseline magnet was questionable in it ability to move the $\mathrm{LO}_{2}$ from the opposite side, by doubling the magnetic field the liquid could be resettled given sufficient time an with $6 x$ the field it resettled quickly.

Based on this initial work, magnetic fields appear to provide a promising means of controlling fluids either independently or by augmenting existing propellant management hardware. The CFD model developed during this activity is a first step in this process, providing a means to evaluate the use of magnetic fields in conjunction with other fluids, hardware devices and tank geometries which more closely represent systems of interest. However, as a final verification of the CFD model's ability, additional test data should be collected and analyzed using $\mathrm{LO}_{2}$ as the test fluid. 